\title{
Tumefactive Demyelination Associated with Bilateral Optic Neuritis in Neuromyelitis Optica Spectrum Disorders
}

\author{
Rajesh Verma ${ }^{1} \quad$ Chetan Kumar $^{1}$ \\ ${ }^{1}$ Department of Neurology, King George's Medical University, \\ Lucknow, Uttar Pradesh, India
}

\begin{abstract}
Address for correspondence Rajesh Verma, DM, DNB, Department of Neurology, King George's Medical University, Lucknow 226003, Uttar Pradesh, India (e-mail: drrajeshverma32@yahoo.com).
\end{abstract}

\author{
Abstract \\ Keywords \\ - tumefactive \\ demyelination \\ - neuromyelitis optica \\ - optic nerves \\ - area prostrema \\ syndrome \\ - tumor \\ - NMO antibody
}

Tumefactive demyelination is an uncommon neurological disorder mimicking tumors. It is one of the rare varieties of demyelinating disorders, often causing diagnostic dilemma among neuroscientists. The literature tells us about approaching these patients added by peculiar neuroimaging findings. Neuromyelitis optica is an immune mediated inflammatory clinical disorder, typically involving optic nerves bilaterally and longitudinally extensive transverse myelitis. With the revelation of aquaporin four channels, its distribution in the brain and related antibody, the concept of neuromyelitis optica spectra disorders has been evolved. In this case report, our intention is to present a young female who presented with bilateral vision loss with tumor-like mass lesion in cerebral cortex. Such an association of bilateral optic neuropathy involving chiasmatic region, suggestive of neuromyelitis optica spectrum disorder (NMOSD) with tumefactive demyelination is rarely reported in the literature.

\section{Introduction}

Tumefactive demyelination is a rare neurological disorder presenting as tumor with demyelinating etiology. Previously it was designated as Schilder's disease or Marburg's variant of multiple sclerosis. ${ }^{1}$ With evolution of research related to demyelinating disorders, it is now specific nosological entity with distinct clinical features and peculiar neuroimaging findings. The tumor-like presentation creates diagnostic dilemma and cause of debate between neurologist and neurosurgeons. The invasive surgery can lead to significant morbidity to the patients. The differentiation between true tumor and tumefactive demyelination is often possible on clinical grounds and with help of neuroimaging. ${ }^{2}$

Neuromyelitis optica spectra disorders have been defined as inflammatory demyelinating immune mediated clinical disorder that mainly involved optic nerves and spinal cord. The involvement can occur either successively or in sequential manner. The myelopathy represents longitudinally extensive transverse myelitis (LETM) and optic neuropathy leads to disabling vision loss. ${ }^{3}$ The concurrence with other autoimmune disorders like systemic lupus erythematosus, Sjogren's syndrome, membranous nephropathy, and hemolytic anemia has been reported in the literature. ${ }^{4}$

In this case report, we presented a young female who manifested as severe bilateral vision loss along with tumefactive demyelination corroborated by neuroimaging. Such association has been scarce in the scientific literature.

\section{Case Presentation}

A 24-year-old female was admitted under department of neurology with history of bilateral visual loss. The patient was apparently asymptomatic 1 month ago, following which she developed pain involving both eyes. It was deep boring pain that was initially more severe in the right eye and persisted throughout the day. Within 1 day of pain onset, she developed loss of vision that initially started with blurring. It involved right eye at the onset. Within 1 week, both her eyes were involved, and she could not even perceive light.

She also had headache along with visual symptoms. Diffuse bifrontal headache persisted throughout the day. 
Occasionally, she woke up from sleep because of headache. It was also associated with vomiting. There was no history of focal neurological deficits involving any body part. The sensory system was intact and did not reveal any abnormality. The autonomic system was unremarkable. The history was negative for joint pains and recurrent abortions.

She had total visual loss, involving both her eyes (no perception of light). Both the pupils were dilated and not reactive to light. Fundus examination revealed pale optic disc with intact disc margins suggestive of primary optic atrophy (-Fig. 1E). There was no restriction of eye movements. There was no evidence of involvement of any other cranial nerves. Motor examination did not reveal hemiparesis. The cerebellar functions were intact.

Investigations: complete blood count, blood sugar level, renal hepatic, and thyroid function tests were within normal limits. Cerebrospinal fluid (CSF) analysis revealed normal range of cells, sugar, protein, and also tested negative for oligoclonal bands. Blood tests for antiaquaporin-4 antibody, serum angiotensin-converting enzyme (ACE) levels, antinuclear antibody (ANA), extracted nuclear antibody (ENA), and antiphospholipid antibody (APLA) were also normal. X-ray of chest and ultrasound study of abdomen were unremarkable.

Magnetic resonance imaging (MRI) studies of brain and spinal cord were done. MRI brain was suggestive of tumefactive demyelination (mass lesion involving right occipitoparietal region; - Fig. 1A, B) with post contrast enhancement involving both optic nerves and optic chiasma (- Fig. 1C, D). Glioma was another close differential diagnosis. Her spinal MRI was found to be normal. Based on this clinical profile and investigative results, the diagnosis of neuromyelititis optica spectra disorder was entertained.

During the course of admission, based on her brain imaging, she was treated with intravenous (IV) methyl prednisolone $1 \mathrm{~g}$ for 5 days. She was also advised immunomodulator agent azathioprine $1 \mathrm{mg} / \mathrm{kg}$ body weight with strict monitoring of liver function test, renal function test, and hematological parameters.

Her brain MRI was repeated after 6 months. It revealed significant reduction in size of the mass lesion which further supported the diagnosis of tumefactive demyelination as component of neuromyelitis optica spectrum disorder (NMOSD; - Fig. 2).

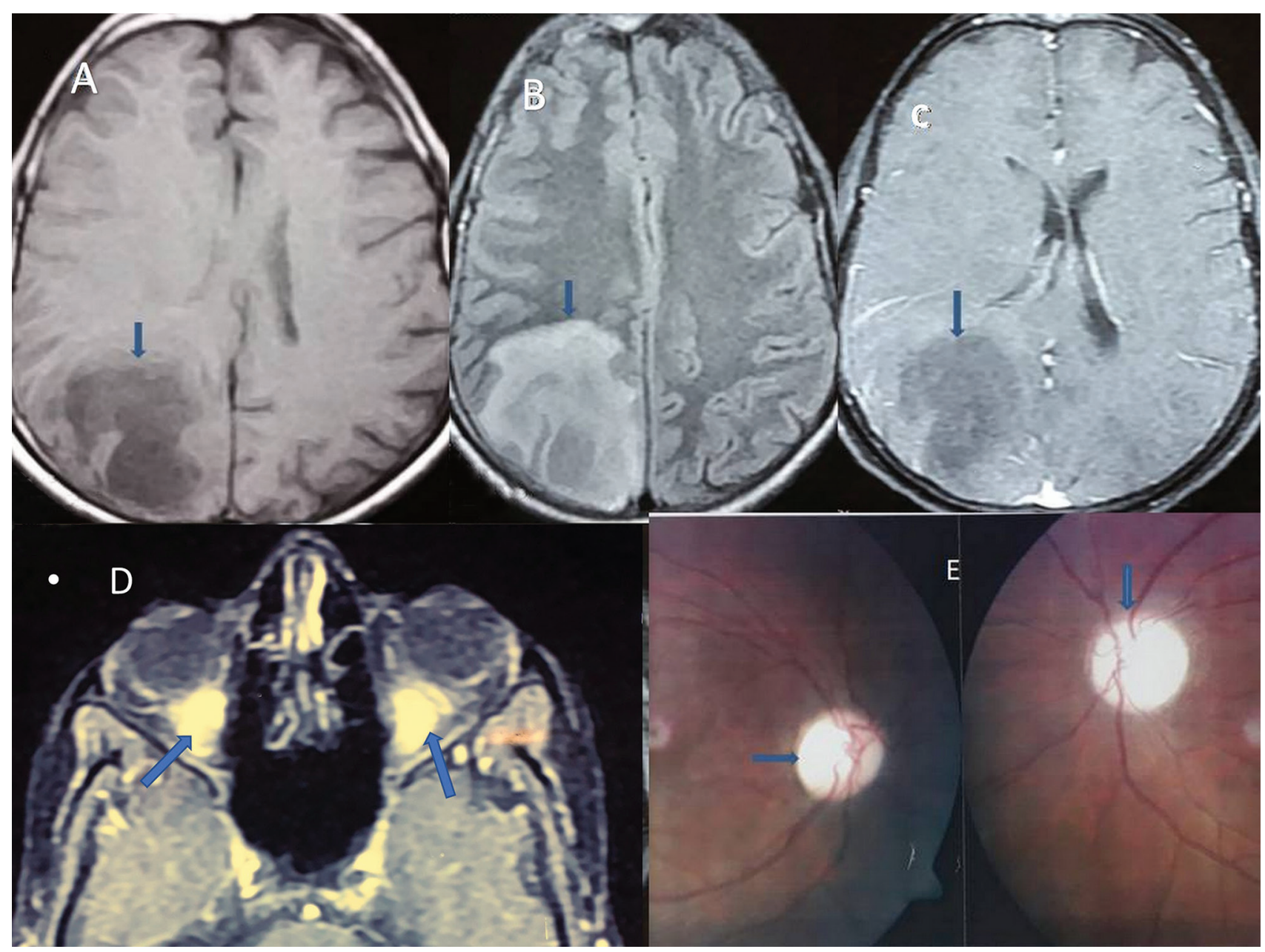

Fig. 1 Magnetic resonance imaging of the cranium (A) heterogeneous lesion in right parietooccipital area on T1-weighed image (B) T2 FLAIRweighed image demonstrating hyperintense mass lesion (vertical blue arrow) (C) showing minimal contrast enhancement with mass effect (arrow on targeted lesion) (D) bilateral optic nerve enhancement till chiasmatic region (arrow indicates the area of optic nerve enhancement). (E) Fundus picture revealed bilateral optic disc atrophy: chalky white disc with intact margins (arrow shows optic disc). FLAIR, fluid attenuated inversion recovery. 


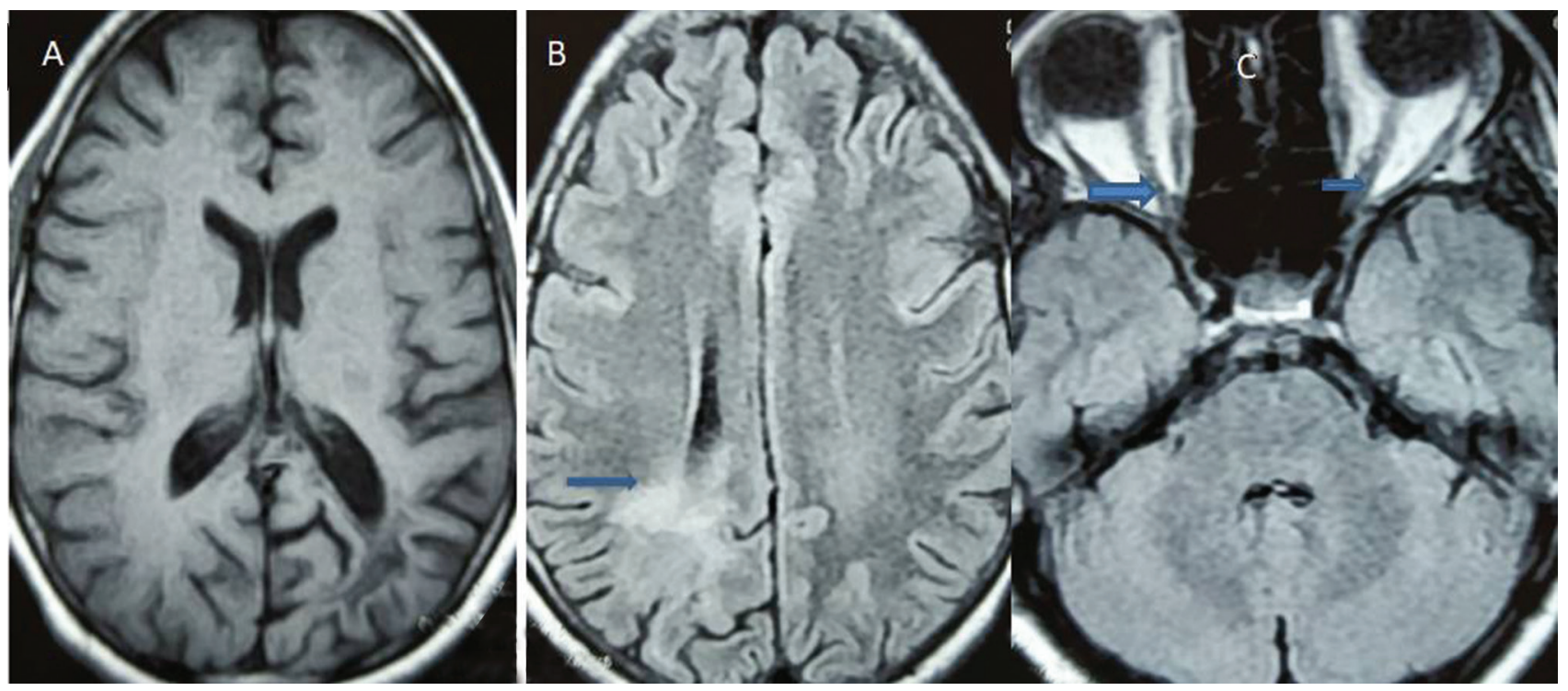

Fig. 2 Post-magnetic resonance images after 6 months (A, B) T1 and T2 FLAIR axial images showed significant resolution of lesion. Arrow indicates resolution of lesion in (B). (C) Repeat MRI with orbit image exhibited well delineated optic nerves (arrow depicts bilateral optic nerves). FLAIR, fluid attenuated inversion recovery; MRI, magnetic resonance imaging.

\section{Discussion}

The demyelinating disorders represents a spectrum of neurological disorders which include multiple sclerosis (MS), acute disseminated encephalomyelitis, neuromyelitis optica, tumefactive demyelination, clinically isolated syndromes, and radiological syndromes. ${ }^{5}$ The distinction among all these syndromes have great clinical implication and having different management strategies. MS is characterized by inflammation and demyelination of brain and spinal cord which often presents as remitting and relapsing type of clinical events, while other variants could be secondary progressive, primary progressive, isolated clinical, and radiological representations. ${ }^{6}$ The MRI findings in MS are of immense help and characteristically lesions are located at periventricular region, perpendicularly as Dawson's fingers, juxtracortical, corpus callosum, brainstem, and spinal cord patchy involvement.

The tumefactive demyelination is well known acceptable clinical entity that behaves like tumor. It presents with varied neurological manifestations. The hemiparesis, impairment in vision, raised intracranial pressure presenting as headache, vomiting, papilleodema, and seizures are important clinical features. ${ }^{8}$ Certain features on neuroimaging are described that suggested tumefactive demyelination. It mainly presents as isolated mass lesion, 2 to $10 \mathrm{~cm}$ in diameter, minimal contrast enhancement, although heterogeneous enhancement has been reported, ring like or half-ring lesions. In early phase, diffusion-weighted imaging may show restriction. Magnetic resonance spectroscopy revealed intact choline to NAA ( $\mathrm{N}$-acetyl aspartate) ratio, while on the contrary the impaired ratio favors presence of tumors. ${ }^{9}$

Our patient revealed minimally enhancing well-circumscribed mass lesion in left sided parietooccipital area. The neuroimaging findings are compatible with diagnosis of tumefactive demyelination. This young female had predominant complaint of persistent bilateral vision loss that was nonresponsive to steroids.
Neuromyelitis optica initially was thought of specific inflammatory-demyelinating disorder involving optic nerves, spinal cord, or either of the two at single point of time. But with the advent of specific NMO antibody (aqaporin-4 antibody), the nomenclature of neuromyelitis optica spectra disorders has been designated. The various distinct syndromes described in NMOSD are longitudinally extensive myelitis, optic neuropathy, area prostrema syndrome, hypothalamic manifestations, and perisylvian lesions. These areas are rich in aquaporin-4 water channels. ${ }^{3,10}$

It is always important to differentiate between NMO and MS as management protocols are entirely different. MS is a relatively progressive demyelinating disorder with predominantly white matter involvement with secondary axonal damage. The lesions are patchy resulting in mild to moderate deficits with good response to immunomodulators. On the other side, NMOSD have discrete lesions, involvement of specific areas of the brain rich in aquaporin water channels, and relatively bad prognosis. ${ }^{11}$

Our case has tumefactive demyelination in the settings of NMOSD. The NMO antibody was negative. The NMO antibody has sensitivity of $68 \%$ and specificity of $86 \%$. Recent criteria categorically state that NMOSD can be considered, even in the absence of NMO antibody, provided clinical phenotypes are compatible with NMO disorders. ${ }^{3,12}$

\section{Conclusion}

This case has been reported because of its rarity. The tumefactive demyelination associated with extensive bilateral optic neuropathy is very unusual and needs attention from treating physicians to manage these types of patients.

\section{Funding}

None. 


\section{Conflict of Interest}

None declared.

\section{References}

1 Giang DW, Poduri KR, Eskin TA, et al. Multiple sclerosis masquerading as a mass lesion. Neuroradiology 1992;34(2):150-154

2 Lucchinetti CF, Gavrilova RH, Metz I, et al. Clinical and radiographic spectrum of pathologically confirmed tumefactive multiple sclerosis. Brain 2008;131(Pt. 7):1759-1775

3 Wingerchuk DM, Banwell B, Bennett JL, et al; International Panel for NMO Diagnosis. International consensus diagnostic criteria for neuromyelitis optica spectrum disorders. Neurology 2015;85(2):177-189

4 Závada J, Nytrová P, Wandinger KP, et al. Seroprevalence and specificity of NMO-IgG (anti-aquaporin 4 antibodies) in patients with neuropsychiatric systemic lupus erythematosus. Rheumatol Int 2013;33(1):259-263

$5 \mathrm{Hu} \mathrm{W}$, Lucchinetti CF. The pathological spectrum of CNS inflammatory demyelinating diseases. Semin Immunopathol 2009;31(4):439-453
6 Polman CH, Reingold SC, Edan G, et al. Diagnostic criteria for multiple sclerosis: 2005 revisions to the "McDonald Criteria." Ann Neurol 2005;58(6):840-846

7 Matthews L, Marasco R, Jenkinson M, et al. Distinction of seropositive NMO spectrum disorder and MS brain lesion distribution. Neurology 2013;80(14):1330-1337

8 Altintas A, Petek B, Isik N, et al. Clinical and radiological characteristics of tumefactive demyelinating lesions: follow-up study. Mult Scler 2012;18(10):1448-1453

9 Nagappa M, Taly AB, Sinha S, et al. Tumefactive demyelination: clinical, imaging and follow-up observations in thirty-nine patients. Acta Neurol Scand 2013;128(1):39-47

10 Jarius S, Franciotta D, Paul F, et al. Testing for antibodies to human aquaporin- 4 by ELISA: sensitivity, specificity, and direct comparison with immunohistochemistry. J Neurol Sci 2012;320(1,2):32-37

11 Pandit L, Asgari N, Apiwattanakul M, et al. GJCF International Clinical Consortium \& Biorepository for Neuromyelitis Optica. Demographic and clinical features of neuromyelitis optica: a review. Mult Scler 2015;21(7):845-853

12 Downer JJ, Leite MI, Carter R, Palace J, Küker W, Quaghebeur G. Diagnosis of neuromyelitis optica (NMO) spectrum disorders: is MRI obsolete? Neuroradiology 2012;54(4):279-285 